\title{
GEOMETRIC HEIGHT INEQUALITIES
}

\section{Kefeng: LiU}

\section{The results}

Let $f: X \rightarrow B$ be a fibration of a compact smooth algebraic surface over a compact Riemann surface $B$, denote by $g \geq 2$ the genus of a generic fiber of $f$ and by $q$ the genus of $B$. Let $s$ be the number of singular fibers of $f$ and $\omega_{X / B}$ be the relative dualizing sheaf. Let $C_{1}, \cdots, C_{n}$ be $n$ mutually disjoint sections of $f$, and denote by $D$ the divisor $\sum_{j=1}^{n} C_{j}$. Then the main result we are going to prove in this note is the following Theorem 0.1

Theorem 0.1. If $f$ is not isotrivial and semistable, then

$$
\left(\omega_{X / B}+D\right)^{2}<(2 g-2+n)(2 q-2+s) .
$$

In fact our proof shows that, for any $n$,

$$
\left(\omega_{X / B}+D\right)^{2}=(2 g-2+n)(2 q-2+s) .
$$

if and only if $f$ is isotrivial.

We can derive several corollaries from this theorem. To state the results, we first introduce some notations. Let $k$ be the function field of $B$ and $\bar{k}$ be its algebraic closure. For an algebraic point $P \in X(\bar{k})$, we let $C_{P}$ be the corresponding horizontal curve on $X$. Let

$$
h_{K}(P)=\frac{\omega_{X / B} \cdot C_{P}}{[k(P): k]}, \quad d(P)=\frac{2 g\left(\tilde{C}_{P}\right)-2}{[k(P): k]}
$$

be respectively the geometric height and the geometric logarithmic discriminant of $P$. Here $\widetilde{C}_{P}$ is the normalization of $C_{P}$ and $[k(P): k]=F \cdot C_{P}$, where $F$ is a generic fiber of $f$, is the degree of $P$. Let $b_{P}$ be the number of ramification points on $\tilde{C}_{P}$ of the induced map $r: \tilde{C}_{P} \rightarrow B$. Write $d_{P}=[k(P): k]$. Then we have

Theorem 0.2. If $f$ is semistable and not isotrivial, then

$$
h_{K}(P)<\left(1+\frac{2 g-2}{d_{P}}\right)\left(d(P)+\frac{b_{P}}{d_{P}}+s\right)-\frac{\omega_{X / B}^{2}}{d_{P}} .
$$

Received July 24, 1996.

This work was partially supported by an NSF grant. 
This theorem gives us a corollary about the geometric height inequality which is originally due to Vojta [V].

Corollary 0.3. Given any $\varepsilon>0$, there exists a constant $O_{\varepsilon}(1)$ depending on $\varepsilon, s, g$ and $q$, such that

$$
h_{K}(P) \leq(2+\varepsilon) d(P)+O_{\varepsilon}(1) .
$$

Vojta conjectured that the above inequality holds with $(2+\varepsilon)$ replaced by $(1+\varepsilon)$. By taking $n=1$ in Theorem 0.1 , we get the following geometric height inequality, a weaker version of which was fisrt proved by Tan [Ta]. For simplicity we still assume $f$ is semistable, the general case follows from the semistable reduction trick as used in [Ta].

Corollary 0.4. Assume $f$ is not isotrivial, then

$$
h_{K}(P) \leq(2 g-1)(d(P)+s)-\omega_{X / B}^{2} .
$$

The equality holds if and only if $f$ is isotrivial.

We note that Theorem 0.2 is stronger than the Vojta's $(2+\varepsilon)$ inequality, but it is slightly weaker than the $(1+\varepsilon)$ conjectural height inequality. I hope that a modification of our method can be used to prove the $(1+\varepsilon)$ conjecture. The only trouble comes from bounding the number of intersections of the $n$ chosen different sections in terms of $n$ and the geometric quantities of the fibration. See Sect. 3.

For the long history of height inequalities and the importance of the Vojta conjecture, we refer the reader to [La], Chapter VI, or [V2]. Especially such inequalities immediately imply the Mordell conjecture for functional field.

When $n=0$, Theorem 0.1 has the following straightforward consequence [B], [Ta1],

Corollary 0.5. (Beauville Conjecture) If $B=C P^{1}$ and $f$ is semistable and not isotrivial, then $s \geq 5$.

Recall that $f$ is called a Kodaira fibration, if $f$ is everywhere of maximal rank but not a complex analytic fiber bundle map. Let $c_{1}(X), c_{2}(X)$ denote the first and second Chern classes of $X$. A very interesting consequence of Theorem 0.1 with $n=0$ is the following Chern number inequality. 
Corollary 0.6. If $f$ is a Kodaira fibration, then

$$
c_{1}(X)^{2}<3 c_{2}(X)
$$

For some special Kodaira fibrations, it is proved in [BPV], pp168, that $c_{1}(X)^{2}<\frac{7}{3} c_{2}(X)$. This particularly implies that a Kodaira surface can not be uniformized by a ball. I was told by Tan and Tsai that this has been unknown for a long time.

The method to prove Theorem 0.1 was first used in [Liu] to prove the case of $n=0$. In [Ta1], Corollary 0.5 was proved by establishing a weaker version of the $n=0$ case of Theorem 0.1. Also in [Ta], a weaker version of Corollary 0.4 is proved. His method is algebro-geometric and is completely different from that of [Liu]. The key technique in [Liu] is the Schwarz-Yau lemma $[\mathrm{Y}]$ and the curvature computations of Tromba [Tr], Wolpert $[\mathrm{W}]$ and Jost $[\mathrm{J}]$ for the Weil-Peterson metric on the moduli spaces of curves. Theorem 0.2 follows from Theorem 0.1 and the stablization theorem in $[\mathrm{Kn}]$. The interested reader may compare with the related works by JostYau $[\mathrm{JY}]$ and Kas $[\mathrm{Ka}]$.

\section{Moduli spaces and the Schwarz-Yau lemma.}

We will use the conventions in [W], [W1] for the geometry of moduli space of semistable curves. Let $\mathcal{M}_{g, n}$ be the moduli space of Riemann surfaces of genus $g$ with $n$ punctures. As in [W] or [TZ], we can consider $\mathcal{M}_{g, n}$ as the moduli space of complex structures on a fixed $n$-punctured Riemann surface $R$.

Let $\pi: \mathcal{T}_{g, n} \rightarrow \mathcal{M}_{g, n}$ be the universal curve and $\bar{\pi}: \overline{\mathcal{T}}_{g, n} \rightarrow \overline{\mathcal{M}}_{g, n}$ their Deligne-Mumford compactifications by adding nodal curves. The Poincare metric on each fiber of $\mathcal{T}_{g, n}$, which is a complete metric on the corresponding $n$-punctured Riemann surface with constant curvature -1 , patches together to give a smooth metric on the relative cotangent bundle $\Omega_{\mathcal{T}_{g, n} / \mathcal{M}_{g, n}}$. The push-down of $\Omega_{\mathcal{T}_{g, n} / \mathcal{M}_{g, n}}^{\otimes 2}$ by $\pi$ which we denote by $\pi ! \Omega_{\mathcal{T}_{g, n} / \mathcal{M}_{g, n}}^{\otimes 2}$, is the cotangent bundle of $\mathcal{M}_{g, n}$. Recall that for any point $z \in \mathcal{M}_{g, n}$,

$$
\left.\pi_{!} \Omega_{\mathcal{T}_{g, n} / \mathcal{M}_{g, n}}^{\otimes 2}\right|_{z}=H^{0}\left(R_{z}, \Omega_{z}^{\otimes 2}\right)
$$

where $R_{z}=\pi^{-1}(z)$ and $\Omega_{z}$ denotes the cotangent bundle of $R_{z}$.

The Poincare metric on each fiber induces a natural inner product on $\pi_{!} \Omega_{\mathcal{T}_{g, n} / \mathcal{M}_{g, n}}^{\otimes 2}$, therefore on the tangent bundle of $\mathcal{M}_{g, n}$ which is just the well-known Weil-Peterson metric on $\mathcal{M}_{g, n}$ [W], [TZ]. 
Lemma 1.1. The holomorphic sectional curvature of the Weil-Peterson metric on $\mathcal{M}_{g, n}$ is negative and strictly bounded from above by $-\frac{1}{\pi(2 g-2+n)}$.

Proof. This is basically due to $[\mathrm{W}],[\mathrm{J}]$ and $[\mathrm{Tr}]$. We follow the argument of [W]. Let $\mu_{\alpha}$ be a tangent vector on $\mathcal{M}_{g, n}$. We know that $\mu_{\alpha}$ is represented by a harmonic Beltrami differential [TZ], [W]. Let $d A$ denote the volume element of the Poincare metric on the $n$ punctured Riemann surface. Assume $\mu_{\alpha}$ is a unit vector, then we have $\left\langle\mu_{\alpha}, \mu_{\alpha}>=\int_{R}\left|\mu_{\alpha}\right|^{2} d A=1\right.$ with respect to the induced metric. The computations in $[\mathrm{W}]$ and $[\mathrm{J}]$ actually tells us that the holomorphic sectional curvature of the Weil-Peterson metric is given by

$$
-R_{\alpha \bar{\alpha} \alpha \bar{\alpha}}=-2<\triangle\left|\mu_{\alpha}\right|^{2},\left|\mu_{\alpha}\right|^{2}>
$$

where $\triangle=-2\left(D_{0}-2\right)^{-1}$ with $D_{0}$ the Laplacian of the Poincare metric on the $n$-punctured Riemann surface. Consider the orthogonal decomposition

$$
\left|\mu_{\alpha}\right|^{2}=\sum_{j} \psi_{j}+E
$$

in terms of the eigenfunctions of $D_{0}$ on $R$. Here $E$ is an Eisenstein series which belongs to the continuous spectrum of $D_{0}$, and $\psi_{j}$ is the eigenfunctions of $D_{0}$ with eigenvalue $\lambda_{j}$. Note that $\lambda_{j}<0$ and the continuous spectrum is contained in $\left(-\infty,-\frac{1}{4}\right]$. So $E$ only has negative contribution to the sectional curvature. Let $\psi_{0}$ be the constant function term in the above decomposition, we then have

$$
-R_{\alpha \bar{\alpha} \alpha \bar{\alpha}} \leq 4 \sum_{j} \frac{<\psi_{j}, \psi_{j}>}{\lambda_{j}-2}<-2<\psi_{0}, \psi_{0}>=-2 \psi_{0}^{2} \int_{R} d A .
$$

Since

$$
\int_{R} \psi_{0} d A=\int_{R}\left|\mu_{\alpha}\right|^{2} d A=1 \text { and } \int_{R} d A=2 \pi(2 g-2+n)
$$

we get $\psi_{0}=\frac{1}{2 \pi(2 g-2+n)}$ which is the required result. As in the $n=0$ case in $[\mathrm{W}]$, this upper bound can not be achieved.

Note that the relative cotangent bundle $\Omega_{\mathcal{T}_{g, n} / \mathcal{M}_{g, n}}$ is a line bundle on $\mathcal{T}_{g, n}$. Its extension to $\overline{\mathcal{T}}_{g, n}$ is the universal dualizing sheaf. Let $c_{1}(\Omega)$ denote its first Chern form with respect to the Poincare metric for $n$-punctures Riemann surface. Let $\pi_{*}$ denote the push-down of cohomology class by $\pi$, i.e. the integral along a generic fiber of $\pi$ and $\omega_{W P}$ the Kahler class of the Weil-Peterson metric. The following lemma is also implicitly proved in $[\mathrm{W}],[\mathrm{W} 1]$ or $[\mathrm{TZ}]$. 
Lemma 1.2. On $\mathcal{M}_{g, n}$ we have

$$
\pi_{*}\left(c_{1}(\Omega)^{2}\right)=\frac{1}{2 \pi^{2}} \omega_{W P}
$$

Furthermore as currents $\pi_{*}\left(c_{1}(\Omega)^{2}\right)$ and $\omega_{W P}$ can be extended to $\overline{\mathcal{M}}_{g, n}$, and (1) still holds as an equality of currents on $\overline{\mathcal{M}}_{g, n}$.

Proof. Equality (1) for punctured surface is proved in [TZ], formula (5.3), following the argument of [W], Corollary 5.11.

It is easy to see that the metric on $\Omega_{\mathcal{T}_{g, n} / \mathcal{M}_{g, n}}$ is good in the sense of Mumford, from [W1], pp420, we know that (1) can be extended to $\overline{\mathcal{M}}_{g, n}$, and as currents $c_{1}(\Omega)$ is continuous on $\overline{\mathcal{T}}_{g, n}$, and represents the first Chern class of the universal dualizing sheaf of $\bar{\pi}: \overline{\mathcal{T}}_{g, n} \rightarrow \overline{\mathcal{M}}_{g, n}$. Also $\omega_{W P}$ has very mild sigularity near the compactification divisor $\overline{\mathcal{M}}_{g, n}-\mathcal{M}_{g, n}$, and as current on $\overline{\mathcal{M}}_{g, n}$ it is the limit of smooth positive Kahler forms in its cohomology class in $H^{2}\left(\overline{\mathcal{M}}_{g, n}, Q\right)$.

We will denote by $\bar{\omega}_{W P}$ and respectively $\bar{\pi}_{*}\left(c_{1}(\Omega)^{2}\right)$ the extensions of $\omega_{W P}$ and $\pi_{*}\left(c_{1}(\Omega)^{2}\right)$ to $\overline{\mathcal{M}}_{g, n}$. So Lemma 1.2 tells us that, as currents on $\overline{\mathcal{M}}_{g, n}$, we have

$$
\bar{\pi}_{*}\left(c_{1}(\Omega)^{2}\right)=\frac{1}{2 \pi^{2}} \bar{\omega}_{W P} .
$$

Note that $\mathcal{M}_{g, n}$ is a $V$-manifold [W1]. The following lemma is a slight refinement of a special case of the general Schwarz-Yau lemma in $[Y]$, and the proof is implicit in $[\mathrm{Y}], \mathrm{pp} .201$.

Lemma 1.3. Let $M$ be a complete Riemann surface with curvature bounded from below by a constant $K_{1}$. Let $N$ be an Hermitian $V$-manifold with holomorphic sectional curvature strictly bounded from above by a negative constant $K_{2}$. Then for any non-constant holomorphic map from $M$ to $N$, one has

$$
f^{*} \omega_{N}<\frac{K_{1}}{K_{2}} \omega_{M}
$$

Where $\omega_{M}, \omega_{N}$ denote respectively the Kahler forms of $M$ and $N$.

Proof. All of the computations in $[\mathrm{Y}]$ is done on the domain manifold $M$, so the formula (21) in [Y] still holds even when $N$ is a $V$-manifold. Next by taking lower limit of $\varepsilon \rightarrow 0$ on both sides of the formula (21) in [Y] and then substitute in the bounds for curvatures.

In fact we only need the following integral version of the above inequality

$$
\int_{M} f^{*} \omega_{N}<\frac{K_{1}}{K_{2}} \int_{M} \omega_{M}
$$


Now we come back to the semistable family introduced at the beginning of the paper. By deleting the $n$ sections, we can view $f: X \rightarrow B$ as a family of $n$-punctured Riemann surfaces. Let $c_{1}\left(\Omega_{X / B}\right)$ be the first Chern form of the relative cotangent bundle with respect to the complete Poincare metric on the $n$-punctured Riemann surface. Of course $c_{1}\left(\Omega_{X / B}\right)$ is first computed on the smooth part $X-f^{-1}(S)$, then since the Poincare metric is good in Mumford sense, as in Lemma 2, it can be extended to $X$ as a current and represents the first Chern class of the relative dualizing sheaf of the $n$-punctured family. Let $\omega_{X / B}$ be the relative dualizing sheaf of $f$ considered as a family of compact Riemann surfaces, and $D$ be the divisor of sections. We will also use $\omega_{X / B}$ and $D$ to denote their corresponding first Chern classes. Let $Q$ be the rational number field.

Lemma 1.4. As cohomology classes in $H^{2}(X, Q)$, we have

$$
c_{1}\left(\Omega_{X / B}\right)=\omega_{X / B}+D
$$

Proof. Let $R$ be a Riemann surface with $n$ punctures, and $\bar{R}$ be its compactification. Let $c_{1}(\Omega)_{R}$ be the first Chern form of the cotangent bundle of $R$ with respect to the complete Poincare metric on $R$. Let $c_{1}(\Omega)_{\bar{R}}$ the

first Chern class of the cotangent bundle of $\bar{R}$, then as currents on $\bar{R}$ we obviously have

$$
c_{1}(\Omega)_{R}=c_{1}(\Omega)_{\bar{R}}+\delta
$$

where $\delta$ is the delta-function of the punctures.

Lemma 1.4 is just a family version of this formula.

\section{The proof of Theorem 0.1}

Now we can prove Theorem 0.1. Let $f: X \rightarrow B$ be the family of semistable curves with $n$ mutually disjoint sections $\left\{C_{j}\right\}$. Then $f$ induces a holomorphic map

$$
h: B \rightarrow \overline{\mathcal{M}}_{g, n}
$$

Let $S$ denote the set of points over which the fibers are singular. Then $s$ is just the number of points in $S$. Since $f$ is not isotrivial, from Lemma 1.3 we know that $s \geq 3$ when $q=0$ and $s \geq 1$ when $q=1$ [Liu], otherwise we must have $q>1$. Here recall that $q$ is the genus of $B$. The restriction of $h$ to $B-S$ induces a holomorphic map

$$
h_{0}: B-S \rightarrow \mathcal{M}_{g, n} .
$$

Equip $B-S$ with the complete Poincare metric whose Kahler form we denote by $\omega_{P}$. On $\mathcal{M}_{g, n}$ we have the Weil-Peterson metric $\omega_{W P}$. Recall 
that the Poincare metric has constant curvature -1 . By applying Lemmas $1.1,1.3$ to $h_{0}$, one has

$$
h_{0}^{*} \omega_{W P}<\pi(2 g-2+n) \omega_{P} .
$$

Integrate over $B-S$, we get

$$
\int_{B-S} h_{0}^{*} \omega_{W P}<\pi(2 g-2+n) \cdot 2 \pi(2 q-2+s) .
$$

Now Lemma 1.2 tells us

$$
\int_{B-S} h_{0}^{*} \omega_{W P}=\int_{B} h^{*} \bar{\omega}_{W P}=2 \pi^{2} \int_{B} h^{*}\left(\bar{\pi}_{*} c_{1}(\Omega)^{2}\right)=2 \pi^{2} \int_{B} f_{*}\left(c_{1}\left(\Omega_{X / B}\right)^{2}\right)
$$

where $c_{1}\left(\Omega_{X / B}\right)$ is the first Chern class of the relative dualizing sheaf of $f: X \rightarrow B$ with respect to the Poincare metric for $n$-punctured Riemann surface.

From Lemma 1.4 we get

$$
\int_{B} f_{*}\left(c_{1}\left(\Omega_{X / B}\right)^{2}\right)=\int_{X} c_{1}\left(\Omega_{X / B}\right)^{2}=\left(\omega_{X / B}+D\right)^{2} .
$$

This finishes the proof of Theorem 0.1 .

\section{The proof of Theorem 0.2}

Let $d=d_{P}=[k(P): k]$. We consider the pull-back family by $r: \tilde{C}_{P} \rightarrow$ $B$ which gives us a semistable family $f_{P}: X_{P} \rightarrow \tilde{C}_{P}$. The pull-back of the horizontal curve $C_{P}$ splits into $d$ sections of $f_{P}$. Let $\tilde{S}$ be the union of the set of points in $\tilde{C}_{P}$ where $r$ ramifies and those points where the fibers of $f: X \rightarrow B$ are singular. Then these $d$ sections, which we denote by $\left\{C_{j}\right\}$, intersect only over the fibers above $\tilde{S}$. Let $D$ denote the divisor $\sum_{j} C_{j}$. It is easy to see that the number of points in $\tilde{S}$ is at most $b+d s$. Here recall that $b$ is the number of points where $r$ ramifies and $s$ is the number of singular fibers of $f$.

By using the stablization theorem in $[\mathrm{Kn}]$, Theorem 2.4, we get a family of semistable $d$-punctured curves $\tilde{f}: Y_{P} \rightarrow \tilde{C}_{P}$. The singular fibers of $\tilde{f}$ are those above $S$. There is a fiberwise contraction map $c: Y_{P} \rightarrow X_{P}$ such that the $d$ mutually disjoint sections of $\tilde{f},\left\{\tilde{C}_{j}\right\}$, are mapped to $\left\{C_{j}\right\}$ correspondingly. Let us write $\tilde{D}=\sum_{j} \tilde{C}_{j}$.

By applying Theorem 0.1 to $\tilde{f}$, we get

$$
\left(\omega_{Y_{P} / \tilde{C}_{P}}+\tilde{D}\right)^{2}<(2 g-2+d)(2 \tilde{q}-2+b+d s)
$$

where $\tilde{q}$ denotes the genus of $\tilde{C}_{P}$. 
Lemma 1.6 in $[\mathrm{Kn}]$ tells us that

$$
\left(\omega_{Y_{P} / \tilde{C}_{P}}+\tilde{D}\right)^{2}=\left(\omega_{X_{P} / C_{P}}+D\right)^{2} .
$$

We deduce from this the following inequality

$$
\omega_{X_{P} / C_{P}}^{2}+\sum_{j=1}^{d} \omega_{Y / C_{P}} \cdot C_{j}<(2 g-2+d)(2 \tilde{q}-2+b+d s) .
$$
have

Now $\omega_{X_{P} / C_{P}}^{2}=d \omega_{X / B}^{2}$, and $\omega_{X_{P} / C_{P}} \cdot C_{j}=\omega_{X / B} \cdot C_{P}$. Therefore we

$$
d \omega_{X / B}^{2}+d \omega_{X / B} \cdot C_{P}<(2 g-2+d)(2 \tilde{q}-2+b+d s) .
$$

Divide both sides of the inequality by $d^{2}$, we get

$$
h_{K}(P)<\left(1+\frac{2 g-2}{d}\right)\left(d(P)+\frac{b}{d}+s\right)-\frac{\omega_{X / B}^{2}}{d}
$$

which is exactly Theorem 0.2 .

\section{The proofs of the corollaries}

To prove Corollary 0.3, we take a large integer $N$ such that, when $d \geq N, \frac{4 g-4}{d}<\varepsilon$. The Hurwitz formula tells us that $b<2 \tilde{q}-2$. So we get from Theorem 0.2 , for $d \geq N$

$$
h_{K}(P)<(2+\varepsilon) d(P)+A
$$

where $A=s\left(1+\frac{\varepsilon}{2}\right)-\frac{\omega_{X / B}^{2}}{d}$.

Next we take another large number $L$ such that for any algebraic point $P$ of degree less than $N$, one has $h_{K}(P)<L$. Obviously $L$ depends on $N$, therefore $\varepsilon, s$ and $q$. Then the inequality in Corollary 0.3 holds with $O_{\varepsilon}(1)=A+L$.

To get such $L$, we make a base change of degree $N !, \pi: C \rightarrow B$ and let $\tilde{f}: \tilde{X} \rightarrow C$ be the pull-back fibration. Then for any algebraic point $P$ of degree $d<N, C_{P} \times{ }_{B} C$ gives a section of $\tilde{f}$. By applying Theorem 0.1 to $\tilde{f}$ with $n=1$, we get

$$
h_{K}(P)<(2 g-1)\left(\frac{\tilde{q}-2+\tilde{s}}{N !}\right)-\omega_{X / B}^{2}
$$

where $\tilde{q}$ and $\tilde{s}$ are respectively the genus of $C$ and the number of singular fibers of $\tilde{f}$. Take $L$ to be the number on the left hand side of this inequality, we are done.

Next we recall the following well-known formulas for the Chern numbers of a semistably fibered algebraic surface $f: X \rightarrow B$ :

$$
c_{1}(X)^{2}=\omega_{X / B}^{2}+8(g-1)(q-1), c_{2}(X)=4(g-1)(q-1)+\delta
$$


where $\delta$ is the number of double points on the fibers. With these two formulas we see that Corollary 0.4 follows from Theorem 0.1 by taking $n=1$ while Corollary 0.6 follows by taking $n=0$.

The proof of Corollary 0.5 follows from a result of Beauville [B] and Theorem 0.1 with $n=0$. In fact Beauville proved that $s \geq 4$ and $s=4$ if and only if $\chi\left(O_{X}\right)=1$. On the other hand we have the following inequality

$$
\operatorname{deg} f_{*} \omega_{X / B} \leq\left(4-\frac{4}{g}\right)^{-1} \omega_{X / B}^{2}
$$

proved by Xiao and Harris-Cornalba. Then recall that

$$
\operatorname{deg} f_{*} \omega_{X / B}=\chi\left(O_{X}\right)-(g-1)(q-1) .
$$

By taking $n=0$ in Theorem 0.1, we get

$$
\chi\left(O_{X}\right)+g-1<\frac{g}{2}(-2+s)
$$

which gives $s>4$.

\section{Acknowledgements}

I would like to thank S. Lu, S. Tan, I. Tsai and S. T Yau for helpful discussions.

\section{References}

[BPV] W. Barth, P. Peters, and A. Van de Ven, Compact complex surfaces, SpringerVerlag, 1984.

[B] A. Beauville, Le nombre minimum de fibres singulières d'une courbe stable sur $P^{1}$, Asterisque 86 (1981), 97-108.

[J] J. Jost, Harmonic maps and the geometry of Teichmuller spaces, Ann. Acad. Sci. Fenn. Ser. A, Math. 16 (1991), 13-48.

[JY] J. Jost and S.-T. Yau Harmonic mappings and algebraic varieties over function fields, Amer. J. Math. 115 (1995), 1197-1228.

[Ka] A. Kas On deformations of certain type of irregular algebraic surfaces, Amer. J. Math. 90 (1968), 789-804.

[Kn] F. Knutson The projectivity of the moduli space of stable curves II, Math. Scand. 52 (1983), 161-199.

[La] S. Lang, Number theory III, Encyclopaedia of Mathematical Sciences 60, Spinger-Verlag, 1991.

[Liu] K. Liu, Remarks on the geometry of moduli spaces, Harvard preprint 1991, to appear in Proc. Amer. Math. Soc.

[Ta] S. Tan, Height inequality of algebraic points on curves over functional fields, preprint, 1994.

[Ta1] _ On the invariants of base changes of pencils of curves, II, preprint, 1994.

[Ta2] _ The minimal number of singular fibers of a semistable curves over $P^{1}$, MPI preprint, 1995. 
[Tr] A. Tromba On a natural algebraic affine connection on the space of almost complex structures and the curvature of Teichmuller space with respect to its Weil-Peterson metric, Man. Math. 56 (1986), 475-497.

[TZ] L. Takhtajan and P. Zograf, A local index theorem for families of $\bar{\partial}$-operators on punctured Riemann surfaces and a new Kahler metric on their moduli spaces, Comm. Math. Phys. 137 (1991), 399-426.

[V] P. Vojta, Diophantine inequalities and Arakelov theory in S. Lang, Introduction to Arakelov Theory, (1988), Springer-Verlag, 155-178.

[V1] _ On algebraic points on curves, Comp. Math. 78 (1991), 29-36.

[V2] —_ Diophantine Approximations and value distribution theory, Lec. Notes in Math. 1329, Springer-Verlag, (1987).

[W] S. Wolpert, Chern forms and the Riemann tensor for the moduli space of curves, Invent. Math. 85 (1985), 119-145.

[W1] _ The hyperbolic metric and the geometry of the universal curves, J. Diff. Geom. 31 (1990), 417-472.

[W2] _ Homology theory of moduli space of stable curves, Ann. Math. 118 (1983), 491-523.

[Y] S.-T. Yau, A general Schwarz lemma for Kahler manifolds, Amer. J. Math. 100 (1978), 197-203.

Department of Mathematics, Stanford, Stanford, CA 94305

E-mail address: kefeng@math.stanford.edu 